\title{
NOTE ON NONLINEAR CONTRACTION SEMIGROUPS
}

\author{
ISAO MIYADERA
}

1. Let $X$ be a Banach space and let $\{T(\xi) ; 0 \leqq \xi<\infty\}$ be a family of nonlinear operators from $X$ into itself satisfying the following conditions:

(i) $T(0)=I$ (the identity) and $T(\xi+\eta)=T(\xi) T(\eta)$ for $\xi, \eta \geqq 0$.

(ii) $\|T(\xi) x-T(\xi) y\| \leqq\|x-y\|$ for $x, y \in X$.

(iii) $\lim _{\xi \rightarrow 0+}\|T(\xi) x-x\|=0$ for $x \in X$.

It is clear that for any fixed $x \in X, T(\xi) x$ is strongly continuous in $\xi \geqq 0$. Such a family $\{T(\xi) ; 0 \leqq \xi<\infty\}$ is called a nonlinear contraction semigroup. Then we define the infinitesimal generator $A$ of $\{T(\xi) ; 0 \leqq \xi<\infty\}$ by

$$
A x=\lim _{\delta \rightarrow 0+} A_{\delta} x
$$

whenever the limit exists, where $A_{\delta}=\delta^{-1}(T(\delta)-I)$. We denote the domain of $A$ by $D(A)$.

In case of linear contraction semigroup, it is well known that

$$
T(\xi) x=\lim _{\delta \rightarrow 0+} T\left(\xi ; A_{\delta}\right) x \quad \text { for } x \in X, \xi \geqq 0,
$$

and for each fixed $x \in X$, the convergence is uniform with respect to $\xi$ in every compact subset of $[0, \infty)$, where

$$
T\left(\xi ; A_{\delta}\right)=\exp \left(\xi A_{\delta}\right)
$$

(see [1]). In this case it is clear that $\left\{T\left(\xi ; A_{\delta}\right) ; 0 \leqq \xi<\infty\right\}$ is a linear contraction semigroup and $T\left(\xi ; A_{\delta}\right)$ is continuous in $\xi \geqq 0$ with respect to the uniform operator topology.

In this paper we shall give similar results for the nonlinear case. The theorem is as follows.

THEOREM. Let $\{T(\xi) ; 0 \leqq \xi<\infty\}$ be a nonlinear contraction semigroup.

I. For each $\delta>0$ there exists a nonlinear contraction semigroup $\left\{T\left(\xi ; A_{\delta}\right) ; 0 \leqq \xi<\infty\right\}$ with the infinitesimal generator $A_{\delta}$ $\left(=\delta^{-1}[T(\delta)-I]\right)$ satisfying the following conditions:

(a) For each $x \in X, T\left(\xi ; A_{\delta}\right) x$ is strongly continuously differentiable in $\xi \geqq 0$ and

$$
d T\left(\xi ; A_{\delta}\right) x / d \xi=A_{\delta} T\left(\xi ; A_{\delta}\right) x \quad \text { for } \xi \geqq 0 .
$$

Received by the editors February 13, 1968 and, in revised form, April 9, 1968. 
(b) For each bounded set $B$ in $X$ and $\xi \geqq 0$,

$$
\sup _{x \in B}\left\|T\left(\xi+h ; A_{\delta}\right) x-T\left(\xi ; A_{\delta}\right) x\right\| \rightarrow 0
$$

as $h \rightarrow 0$.

II. Suppose that

(iv) there exists a set $D$ such that $D \subset D(A)$ and for any $x \in D$, $T(\xi) x \in D(A)$ for a.e. $\xi>0$.

Then for each $x \in D^{-}$(the closure of $D$ ) we have

$$
T(\xi) x=\lim _{\delta \rightarrow 0+} T\left(\xi ; A_{\delta}\right) x \quad \text { for } \xi \geqq 0,
$$

and the convergence is uniform with respect to $\xi$ in every compact subset of $[0, \infty)$.

Hence, if $D$ is dense in $X$, then (3) holds good for all $x \in X$.

The proof is given in $\$ 3$.

REMARK. The author found in Notices of the American Mathematical Society that J. R. Dorroh [2] has obtained the same result under the assumption

'for each $x \in D, T(\xi) x$ is strongly continuously differentiable in $\xi \geqq 0$.'

Y. Kömura [4] has proved that if $X$ is a Hilbert space, then for each $x \in D(A)$

(4) $T(\xi) x \in D(A) \quad$ for a.e. $\xi \geqq 0$, and $T(\xi) x=x+\int_{0}^{\xi} A T(\eta) x d \eta$.

(He has shown that (4) holds good for each $x \in D\left(A_{\Phi}\right)$.) It follows from his argument that (4) is also true for reflexive Banach spaces. Hence, if $X$ is a reflexive Banach space, then our assumption (iv) is always satisfied by taking $D=D(A)$. Thus we have the following

CoRollary. If $\{T(\xi) ; 0 \leqq \xi<\infty\}$ is a nonlinear contraction semigroup defined on a reflexive Banach space $X$, then for each $x \in(D(A))^{-}$

$$
T(\xi) x=\lim _{\delta \rightarrow 0+} T\left(\xi ; A_{\delta}\right) x \quad \text { for } \xi \geqq 0,
$$

and the convergence is uniform with respect to $\xi$ in every compact subset of $[0, \infty)$.

Especially, if $D(A)$ is dense in $X$, then the above convergence holds good for all $x \in X$.

REMARK. Y. Kōmura [4] proved that $(D(A))^{-}=X$ if $X=R^{n}$ (the $n$-dimensional Euclidean space). 
2. The proof of the main part II in our theorem is based on the following Kato's lemmas [3].

LEMMA 1. Let $T$ be an operator with domain $D(T)$ and range $R(T)$ in $X$. The following conditions are equivalent:

(D) $\|x-y-\alpha(T x-T y)\| \geqq\|x-y\|$ for every $x, y \in D(T)$ and $\alpha>0$. (That is, $-T$ is monotonic in the sense of Kato.)

$\left(\mathrm{D}^{\prime}\right)$ For each $x, y \in D(T)$, there is $f \in F(x-y)$ such that

$$
\operatorname{Re}(T x-T y, f) \leqq 0,
$$

where $F$ is the duality map from $X$ into $X^{*}$.

Lемма 2. Let $x(\xi)$ be an $X$-valued function on an interval of real numbers. Suppose $x(\xi)$ has a weak derivative $x^{\prime}(\eta) \in X$ at $\xi=\eta$. If $\|x(\xi)\|$ is also differentiable at $\xi=\eta$, then

$$
\|x(\eta)\|[d\|x(\xi)\| / d \xi]_{\xi=\eta}=\operatorname{Re}\left(x^{\prime}(\eta), f\right)
$$

for every $f \in F x(\eta)$.

Since $T(\delta)$ is a contraction and $A_{\delta}=\delta^{-1}[T(\delta)-I]$, we have

$$
\left\|A_{\delta} x-A_{\delta} y\right\| \leqq 2\|x-y\| / \delta \text { for } x, y \in X,
$$

and

$$
\left\|x-y-\alpha\left(A_{\delta} x-A_{\delta} y\right)\right\| \geqq\|x-y\|
$$

for $x, y \in X$ and $\alpha>0$.

Now we shall prove the following

LEMMA 3. The equation

$$
\begin{aligned}
\frac{d u(\xi ; x)}{d \xi} & =A_{\delta} u(\xi ; x) \text { for } \xi \geqq 0 \\
u(0 ; x) & =x
\end{aligned}
$$

has a unique solution $u(\xi ; x) \in C^{1}([0, \infty) ; X)$ for any $x \in X$, and

$$
\|u(\xi ; x)-u(\xi ; y)\| \leqq\|x-y\| \text { for } x, y \in X \text { and } \xi \geqq 0
$$

where $C^{1}([0, \infty) ; X)$ denotes the set of all strongly continuously differentiable $X$-valued functions defined on $[0, \infty)$.

Proof. Since the map $x \rightarrow A_{\delta} x$ is Lipschitz continuous, uniformly in $x$ (see (5)), the equation ( 7 ) has a unique solution $u(\xi ; x)$ $\in C^{1}([0, \infty) ; X)$ for any $x \in X$.

We shall prove (8). Fix $x, y \in X$ and put 


$$
z(\xi)=u(\xi ; x)-u(\xi ; y) .
$$

Clearly $z(\xi) \in C^{\mathbf{1}}([0, \infty) ; X)$ and

$$
\begin{aligned}
d z(\xi) / d \xi & =A_{\delta} u(\xi ; x)-A_{\delta} u(\xi ; y), \\
z(0) & =x-y .
\end{aligned}
$$

Since $\|z(\xi)\|$ is absolutely continuous, $\|z(\xi)\|$ is differentiable for a.e. $\xi \geqq 0$. Therefore, by Lemma 2 , we get for a.e. $\eta \geqq 0$

$$
\begin{aligned}
\|z(\eta)\|[d\|z(\eta)\| / d \eta] & =\operatorname{Re}\left(z^{\prime}(\eta), f_{\eta}\right) \\
& =\operatorname{Re}\left(A_{\delta} u(\eta ; x)-A_{\delta} u(\eta ; y), f_{\eta}\right)
\end{aligned}
$$

for every $f_{\eta} \in F z(\eta)=F(u(\eta ; x)-u(\eta ; y))$. Therefore it follows from (6) and Lemma 1 that

$$
\|z(\eta)\|[d\|z(\eta)\| / d \eta] \leqq 0 \quad \text { for a.e. } \eta \geqq 0 .
$$

Since

$$
\begin{aligned}
\|z(\xi)\|^{2}-\|z(0)\|^{2} & =\int_{0}^{\xi}\left[d\|z(\eta)\|^{2} / d \eta\right] d \eta \\
& =2 \int_{0}^{\xi}[\|z(\eta)\| \cdot d\|z(\eta)\| / d \eta] d \eta \leqq 0
\end{aligned}
$$

for each $\xi>0$, we obtain

$$
\|u(\xi ; x)-u(\xi ; y)\| \leqq\|x-y\| \quad \text { for } \xi>0 . \quad \text { Q.E.D. }
$$

REMARK. It is possible to prove the inequality (8) without using the duality map (that is Lemmas 1 and 2).

\section{Proof of the theorem.} let

Proof of I. Let $u(\xi ; x)$ be the (unique) solution in Lemma 3 , and

$$
T\left(\xi ; A_{\delta}\right) x=u(\xi ; x) \quad \text { for } \xi \geqq 0 \text { and } \quad x \in X .
$$

It is clear that $\left\{T\left(\xi ; A_{\delta}\right) ; 0 \leqq \xi<\infty\right\}$ is a nonlinear contraction semigroup satisfying the condition (a).

We shall show (b).

$$
\left\|T\left(\xi+h ; A_{\delta}\right) x-T\left(\xi ; A_{\delta}\right) x\right\|=\left\|\int_{\xi}^{\xi+h} A_{\delta} T\left(\eta ; A_{\delta}\right) x d \eta\right\|
$$

$$
\leqq\left|\int_{\xi}^{\xi+h}\left\|A_{\delta} T\left(\eta ; A_{\delta}\right) x\right\| d \eta\right| \leqq|h|\left\|A_{\delta} x\right\|
$$


because $\left\|A_{\delta} T\left(\eta ; A_{\delta}\right) x\right\| \leqq\left\|A_{\delta} x\right\|$. If $B$ is a bounded set, then $\sup _{x \in B}\left\|A_{\delta} x\right\|\left(=M_{B}\right)<\infty$; hence, by $(10)$, we have

$$
\sup _{x \in B}\left\|T\left(\xi+h ; A_{\delta}\right) x-T\left(\xi ; A_{\delta}\right) x\right\| \leqq M_{B}|h| \rightarrow 0
$$

as $h \rightarrow 0$.

Proof of II. Now assume

(iv) there exists a set $D$ such that $D \subset D(A)$ and for each $x \in D$, $T(\xi) x \in D(A)$ for a.e. $\xi>0$.

Let $x \in D$.

(11) $A T(\xi) x=\lim _{h \rightarrow 0+} \frac{T(h)-I}{h} T(\xi) x=\lim _{h \rightarrow 0+} \frac{T(\xi+h) x-T(\xi) x}{h}$

for a.e. $\xi \geqq 0$; and

$$
\|A T(\xi) x\| \leqq\|A x\| \quad \text { for a.e. } \xi \geqq 0,
$$

since $\left\|h^{-1}[T(\xi+h) x-T(\xi) x]\right\| \leqq\left\|h^{-1}[T(h) x-x]\right\|$. Hence $A T(\xi) x$ is strongly measurable and bounded, and so $A T(\xi) x$ is Bochner integrable on any finite interval. Let $f \in X^{*}$. It is easy to see that $T(\xi) x$ is strongly absolutely continuous in $\xi$ and a fortiori $(T(\xi) x, f)$ $(=f(T(\xi) x))$ is absolutely continuous. Hence $(T(\xi) x, f)$ is differentiable at a.e. $\xi \geqq 0$ and

$$
(T(\xi) x, f)-(x, f)=\int_{0}^{\xi} \frac{d}{d \eta}(T(\eta) x, f) d \eta
$$

for all $\xi \geqq 0$. Moreover, it follows from (11) that

$$
(d / d \eta)(T(\eta) x, f)=(A T(\eta) x, f) \quad \text { for a.e. } \eta \geqq 0 .
$$

Thus the above equalities and the Bochner integrability of $A T(\xi) x$ show that

$$
\begin{aligned}
(T(\xi) x, f)-(x, f) & =\int_{0}^{\xi}(A T(\eta) x, f) d \eta \\
& =\left(\int_{0}^{\xi} A T(\eta) x d \eta, f\right) \quad \text { for all } \xi \geqq 0 .
\end{aligned}
$$

Hence we obtain

$$
T(\xi) x-x=\int_{0}^{\xi} A T(\eta) x d \eta \quad \text { for all } \xi \geqq 0,
$$

and then $(d / d \xi) T(\xi) x=A T(\xi) x$ for a.e. $\xi \geqq 0$. 
If we put

$$
z_{\delta}(\xi)=T\left(\xi ; A_{\delta}\right) x-T(\xi) x \quad\left(=\int_{0}^{\xi}\left[A_{\delta} T\left(\eta ; A_{\delta}\right) x-A T(\eta) x\right] d \eta\right)
$$

for $\xi \geqq 0$, then $z_{\delta}(\xi)$ has strong derivative

$$
z_{\delta}^{\prime}(\xi)=A_{\delta} T\left(\xi ; A_{\delta}\right) x-A T(\xi) x \quad \text { for a.e. } \xi \geqq 0,
$$

and $\left\|z_{\delta}(\xi)\right\|$ is also differentiable for a.e. $\xi \geqq 0$ since $\left\|z_{\delta}(\xi)\right\|$ is absolutely continuous with respect to $\xi$. Using Lemma 2 , for a.e. $\xi \geqq 0$

$$
\begin{aligned}
\left\|z_{\delta}(\xi)\right\|\left[d\left\|z_{\delta}(\xi)\right\| / d \xi\right] & =\operatorname{Re}\left(z_{\delta}^{\prime}(\xi), f_{\xi}\right) \\
& =\operatorname{Re}\left(A_{\delta} T\left(\xi ; A_{\delta}\right) x-A T(\xi) x, f_{\xi}\right)
\end{aligned}
$$

for every $f_{\xi} \in F z_{\delta}(\xi)=F\left(T\left(\xi ; A_{\delta}\right) x-T(\xi) x\right)$. Moreover, by (6) and Lemma 1 , for each $\xi \geqq 0$ there is $f_{\xi}^{0} \in F z_{\delta}(\xi)=F\left(T\left(\xi ; A_{\delta}\right) x-T(\xi) x\right)$ such that

$$
\operatorname{Re}\left(A_{\delta} T\left(\xi ; A_{\delta}\right) x-A_{\delta} T(\xi) x, f_{\xi}^{0}\right) \leqq 0 .
$$

Combining this and (13), we obtain

$$
\left\|z_{\delta}(\xi)\right\|\left[d\left\|z_{\delta}(\xi)\right\| / d \xi\right] \leqq \operatorname{Re}\left(A_{\delta} T(\xi) x-A T(\xi) x, f_{\xi}^{0}\right)
$$

for a.e. $\xi \geqq 0$; and hence

$$
\begin{aligned}
\left\|z_{\delta}(\xi)\right\|\left[d\left\|z_{\delta}(\xi)\right\| / d \xi\right] & \leqq\left\|A_{\delta} T(\xi) x-A T(\xi) x\right\|\left\|f_{\xi}^{0}\right\| \\
& =\left\|A_{\delta} T(\xi) x-A T(\xi) x\right\|\left\|T\left(\xi ; A_{\delta}\right) x-T(\xi) x\right\|
\end{aligned}
$$

for a.e. $\xi \geqq 0$. Thus for each $\xi \geqq 0$

$$
\begin{aligned}
\left\|z_{\delta}(\xi)\right\|^{2} & =\int_{0}^{\xi}\left[d\left\|z_{\delta}(\eta)\right\|^{2} / d \eta\right] d \eta=2 \int_{0}^{\xi}\left\|z_{\delta}(\eta)\right\|\left[d\left\|z_{\delta}(\eta)\right\| / d \eta\right] d \eta \\
& \leqq 2 \int_{0}^{\xi}\left\|A_{\delta} T(\eta) x-A T(\eta) x\right\|\left\|T\left(\eta ; A_{\delta}\right) x-T(\eta) x\right\| d \eta
\end{aligned}
$$

so that for any $\beta>0$

$$
\begin{aligned}
\sup _{0 \leqq \xi \leqq \beta} \| T(\xi ; & \left.A_{\delta}\right) x-T(\xi) x \|^{2} \\
& \leqq 2 \int_{0}^{\beta}\left\|A_{\delta} T(\eta) x-A T(\eta) x\right\|\left\|T\left(\eta ; A_{\delta}\right) x-T(\eta) x\right\| d \eta
\end{aligned}
$$

We have 


$$
\begin{gathered}
\lim _{\delta \rightarrow 0+}\left\|A_{\delta} T(\eta) x-A T(\eta) x\right\|=0 \quad \text { for a.e. } \eta \geqq 0, \\
\|A T(\eta) x\| \leqq\|A x\| \quad \text { for a.e. } \eta \geqq 0, \\
\left\|A_{\delta} T(\eta) x\right\| \leqq\left\|A_{\delta} x\right\| \quad(=O(1) \text { as } \delta \rightarrow 0+) \quad \text { for all } \eta \geqq 0,
\end{gathered}
$$

and

$$
\begin{aligned}
\left\|T\left(\eta ; A_{\delta}\right) x\right\| & \leqq\|x\|+\int_{0}^{\eta}\left\|A_{\delta} T\left(\tau ; A_{\delta}\right) x\right\| d \tau \\
& \leqq\|x\|+\left\|A_{\delta} x\right\| \beta \quad \text { for } \eta \in[0, \beta] .
\end{aligned}
$$

These show that the integrand of (15) converges boundedly to zero as $\delta \rightarrow 0+$. Thus it follows from the convergence theorem that for any $\beta>0$

$$
\sup _{0 \leqq \xi \leqq \beta}\left\|T\left(\xi ; A_{\delta}\right) x-T(\xi) x\right\| \rightarrow 0
$$

as $\delta \rightarrow 0+$.

Finally, since $T\left(\xi ; A_{\delta}\right)$ and $T(\xi)$ are contraction operators, it is clear that (16) holds good for each $x \in D^{-}$. Q.E.D.

The author wishes to thank the referee for many helpful comments.

Added in Proof. Recently the author proved the following: "If $C$ is a contraction operator (not necessarily linear) from $X$ into itself, then $\left\|T(m ; C-I) x-C^{m} x\right\| \leqq m^{1 / 2}\|(C-I) x\|$ for every $x \in X$ and positive integer $m$, where $\{T(\xi ; C-I) ; 0 \leqq \xi<\infty\}$ is a nonlinear contraction semigroup generated by $C-I$."

In the case of linear operators, this has been obtained by P. R. Chernoff (Note on product formulas for operator semigroups, J. Functional Analysis 2 (1968), 238-242).

Then it follows from the above result that, without the assumption (iv), the convergence (3) in the theorem holds for each $x \in D(A)^{-}$ (i.e., the corollary remains true for general Banach space $X$ ).

\section{BiBLIOGRAPHY}

1. E. Hille and R. S. Phillips, Functional analysis and semigroups, Amer. Math. Soc. Colloq. Publ., Vol. 31, Amer. Math. Soc., Providence, R.I., 1957.

2. J. R. Dorroh, Semi-groups of nonlinear transformations, Abstract 653-171, Notices Amer. Math. Soc. 15 (1968), 128.

3. T. Kato, Nonlinear semi-groups and evolution equations, J. Math. Soc. Japan 19 (1967), 508-520.

4. Y. Kōmura, Nonlinear semi-groups in Hilbert space, J. Math. Soc. Japan 19 (1967), 493-507.

WASEDA UNIVERSITY AND GEORGETOWN UNIVERSITY 